\title{
EVALUATION OF INJECTABLE PLATELET RICH FIBRIN FOR THE MANAGEMENT OF TEMPROMANDIBULAR JOINT INTERNAL DERANGEMENT. (CLINICAL EVALUATION)
}

\author{
Usama Abdeltawab Teama * and Hesham S. Abdelmoneim*
}

\begin{abstract}
Temporomandibular joint (TMJ) disorders are utterly described by joint dysfunction and/or pain. Temporomandibular joint internal derangement is the most frequently reported temporomandibular disorder. Several methods have been used for the management of TMJ internal derangement ranging from conservative occlusal splints and physiotherapy, to minimally invasive arthrocentesis and to open joint invasive surgical procedures. All of which aim to restore function and relief affected joints pain. Current study aimed on clinically evaluating the effect of using injectable platelet rich fibrin as an injection drug in the temporomandibular joint following arthrocentesis procedure. Results of our study revealed that the injectable PRF group was superior to the normal arthrocentesis group in pain relief, rapidity and amount of regain of the maximum mouth opening and in the resolution of the joint noise. Concluding that intra-articular PRF injection of the TMJ is a significantly effective method for the treatment of internal derangement.
\end{abstract}

Key words; arthrocentesis, injectable PRF, TMJ internal derangement

\section{INTRODUCTION}

Temporomandibular joint (TMJ) disorders are usually characterized by joint dysfunction and/or pain. Temporomandibular joint internal derangement (ID) is the most frequently reported temporomandibular disorder (TMD), reaching approximately $41.1 \%$ of individuals suffering from TMDs. The internal derangement of the TMJ was defined as joint dysfunction associated with an abnormal articular disc position. Successful treatment of the TMD depends on meticulous and proper Identification and elimination of the etiologic factors that result in TMJ tissue breakdown. Among these causes are Para-functional habits and trauma that would lead to degenerative alterations in the articular tissues that in turn leads to increased friction and gradual disc affection. Systemic degenerative arthropathies could also affect the TMJ and cause internal derangement. ${ }^{(1)}$

* Lecturer of Oral and Maxillofacial Surgery, Faculty of Dentistry, Cairo University. 
Internal derangement has been vastly defined as "joint dysfunction associated with an abnormal disc position and damage to the internal structures of the joint" (1). Among the most updated guidelines and classification systems are Wilkes staging ${ }^{(2)}$ and Diagnostic Criteria for TMDs ${ }^{(3)}$.

The Wilkes staging system for Internal derangement of TMJ is vastly used by oral and maxillofacial specialists to provide a guide for treatment based upon the severity of the internal joint damage ( Table 1 ). The Diagnostic Criteria /TMD classification system has been vigorously tested (in terms of sensitivity and specificity) and validated for various linguistics, the current update for DC/TMD was out in 2014, it is composed of two axes 1 and 2, where AXIS 1 for classification of the physical categories of the TMDs, and AXIS 2 for classification of psychosocial behavioral aspects of individuals with TMDs. Accordingly Axis 1 divided TMJ internal derangement into four stages: disc displacement with reduction, disc displacement with reduction with intermittent locking, disc displacement without reduction with limitation in mouth opening and disc displacement without reduction without limitation in mouth opening. The major drawback of these classification systems is that there are no etiological factors associated with the corresponding stage, although understanding the actual cause for joint structures damage is crucial for the proper treatment, which in turn could be the cause of treatment failure due to the persistence of the causative factors. ${ }^{(1-3)}$

Management is mostly focused on relieving the functional pain and re-establishing normal range of mandibular function. Management includes noninvasive methods like occlusal splints, supportive physical therapy, rehabilitation involving muscular training, and even psychological support, all of which aiming to relieve the symptoms (4). However, if symptoms remain and the severity intensified minimally invasive treatments might be considered, including lavage (arthrocentesis) with or without intra- articular injections of hyaluronic acid, corticosteroids, or chondroitinsulphate and arthroscopy ${ }^{(5,6)}$.

Several researches aimed on utilizing methods to stimulate repair or even replacement of damaged cartilage, as matrix metalloproteinase inhibitor, cytokine inhibitor, gene therapy, artificial cartilage substitutes and growth factors. Autologous Platelets Concentrates contains growth factors as (Platelet derived growth factor, transforming growth factor $\beta$, vascular endothelial growth factor), endostatins, platelet factor 4 , thrombospondin and angiopoietins. Thereby these intra-articular injections reduces inflammation, relief pain, improve mandibular function and could stimulate cartilage regeneration. Analgesic potential of platelets are thought to be due to the release of protease activated receptor 4 peptides. ${ }^{(7,8)}$

TABLE (1) Showing Wilkes staging system.

\begin{tabular}{|l|l|l|}
\hline Stage & Clinical findings & Radiological findings \\
\hline I (Early) & Painless click, no limitation in motion & $\begin{array}{l}\text { Slight disc displacement with early reduction, normal disc } \\
\text { morphology }\end{array}$ \\
\hline II (Early/intermediate) & $\begin{array}{l}\text { Occasional painful click, intermittent } \\
\text { locking, related headache }\end{array}$ & $\begin{array}{l}\text { Moderate disc displacement with late reduction, mild disc } \\
\text { deformity }\end{array}$ \\
\hline III (Intermediate) & $\begin{array}{l}\text { Frequent pain, joint tenderness, } \\
\text { restricted motion, closed lock }\end{array}$ & $\begin{array}{l}\text { Disc displacement without reduction, deformity of the disc, } \\
\text { no hard tissue changes. }\end{array}$ \\
\hline IV (Intermediate/late) & Chronic pain, restriction of motion & $\begin{array}{l}\text { Severe disc displacement (without reduction), Severe } \\
\text { deformity of the disc and degenerative changes. }\end{array}$ \\
\hline V (Late) & $\begin{array}{l}\text { Variable and episodic joint pain, chronic } \\
\text { restriction of motion and crepitus. }\end{array}$ & $\begin{array}{l}\text { Gross deformity and/or perforation of disc, degenerative arthritic } \\
\text { changes, osteophyte deformity with subcortical cyst formation. }\end{array}$ \\
\hline
\end{tabular}


It has been stated by several researchers that platelet-rich plasma was highly efficient in the management of temporomandibular disorders. As the second-generation platelet concentrates known as platelet rich fibrin (PRF) was developed and proved to enhance wound healing compared to PRP an enormous amount of publications and applications has been done to unleash its' potential. As Standard PRF contains a 3-dimensional fibrin matrix that is not suitable for injection there for its applications in the TMDs were limited only to invasive surgical procedure (open joint surgery). ${ }^{(7,9,10)}$

A novel low speed centrifugation method reviled a concept of injectable PRF (i-PRF) that's a liquid formulation of PRF. It maintains a liquid form for several minutes following centrifugation and thereby could be injected. It has been stated that injectable PRF has a high the ability to release large concentrations of various growth factors, induce fibroblastic migration and expression of PDGF, TGF- $\beta$, and collagen 1 thus provide a good environment for the regeneration and repair of the tissues..$^{(11-13)}$

Flowable (injectable) PRF has been stated to exhibit long-term analgesic (pain relief) effects in patients with painful TMJ internal derangement, with promising outcomes despite not being strongly effective on early stages of joint derangement. ${ }^{(14)}$

Injectable PRF has been previously investigated in the TMJ for the management of joint hypermobility by Menerva A. Elgazzaz et al. whose research concluded that PRF injection is an effective, minimally invasive technique for the management of TMJ hypermobility through enhancing capsular and ligamental integrity adding its role in relieving the signs and symptoms. Also it was recommended that further investigation about the benefits of Injectable PRF injection in the Joint for the management of various temporomandibular disorders, all due to the reported and observed improvement both clinical and radiological. ${ }^{(15)}$

Several TMJ arthrocentesis (lavage) materials for the relief of the signs and symptoms of TMJ internal derangement have been previously reported. Among these materials were normal saline, ozonized water and lactated Ringer lavage followed by corticosteroid, sodium hyaluronate, platelet rich plasma (PRP) and ozone injections. All with variable results and no optimum material could be concluded. ${ }^{(16-18)}$

PRF could be considered as fibrin meshwork of nano-scale fibers that acts as a scaffold favoring cell migration, differentiation and proliferation, also it could be considered as a drug delivery system of growth factors, which could enhance healing and stimulate neo-angiogenesis. There by PRF has been widely applied and investigated in various dental and surgical fields with promising results. And with the rise the modified preparation method of PRF and development of flowable or injectable PRF the applications were widely augmented based on its reported increase release of growth factors and angiogenesis, suggesting injectable PRF a highly beneficial therapeutic medium for tissue engineering. ${ }^{(19-21)}$

From the documented benefits and advantages of injectable PRF our study aimed to evaluate the possible positive outcome of the use of injectable PRF as an intra-articular injection solution in arthrocentisis (joint lavage) procedures for the management of internal derangement of the TMJ.

\section{METHODOLOGY}

This study was a randomized, double blinded, controlled clinical trial where patients enrolled in this study were allocated into 2 groups. Patients enrolled in this study had to fall under certain inclusion criteria;

- Patients should be free from any systemic condition that might alter the predicted outcomes, or even contraindicate the surgical intervention.

- Patients suffering from internal derangement of their TMJ (unilateral or bilateral) stages II, III and IV Wilkes staging system. 
- Patients should be first undergone a non invasive management of their derangement by occlusal splints that yielded to be insufficient for the relief of their signs and symptoms before being enrolled in this study.

This study was conducted on 30 patients into which 36 TMJs were operated on. The patients were divided into two groups, A (control); into which the affected joints received arthrocentesis (lavage) with regular saline solution ( $\mathrm{NaCL} 0,9 \%$ ). Where two needle technique was followed, in which the affected joint was lavaged with $300 \mathrm{ml}$ of saline solution concomitant with vigorous manipulation of the mandible to ensure full range of mandibular motion and release of any adhesions and free the adhered disc if present. While in group B (intervention) the enrolled joints were lavaged by the same technique as group $\mathrm{A}$ and then injected with injectable (flowable) PRF shortly after its preparation.

Injectable PRF was prepared from $20 \mathrm{ml}$ of blood withdrawn from the cubital vein of the enrolled patient, the withdrawn blood was then divided into two $10 \mathrm{ml}$ vaccutainers (plain tubes) then centrifuged for two minutes at $3300 \mathrm{rpm}^{(22)}$, the obtained injectable PRF (fig. 1) was then aspirated from the vaccutainers in a $5 \mathrm{ml}$ plastic syringe and 1.5 to $2 \mathrm{ml}$ of PRF were then injected into the superior joint space of the lavaged joints (fig. 2).

Superior repositioning splints (SRS) were fabricated for all the our cases to be used post operatively. Patients were prescribed with analgesics for the control of pain post-operatively.

Patients of both groups were clinically evaluated by measuring the pre-operative maximum mouth (inter inscisal) opening (MMO) (fig.3) and compared with the MMO 1week, 2 weeks, 8 weeks post-operative. Patients' pain was assessed by visual analogue scale per operative and 1,2 weeks, 8 weeks post-operative. Auscultation of the joints was performed to monitor the persistence or relief of the joint noise (clicking) at the same intervals in cases with reported preoperative click.
Results were statistically analized via Microsoft excel statistical analyzer, AVERAGE, STDEV and $\mathrm{P}$ value calculated by $\mathrm{T}$ test to compare variables between the two groups. The results were considered statistically significant if the $\mathrm{p}$ value was $\leq 0.05$.

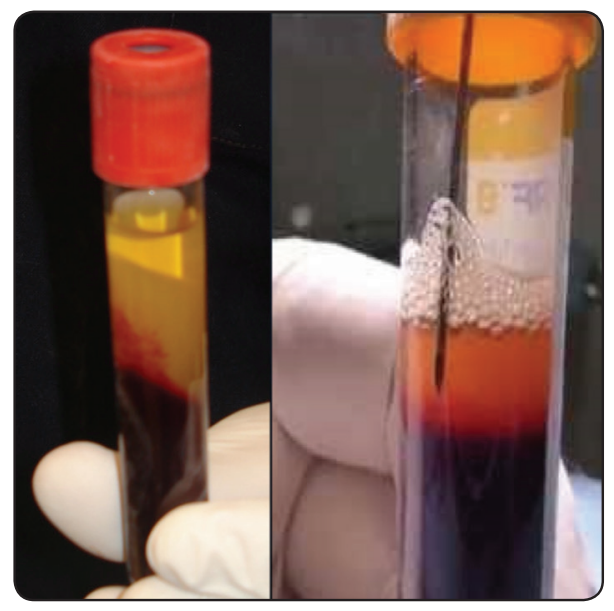

Fig. (1) Showing the obtained injectable PRF after centrifugation.

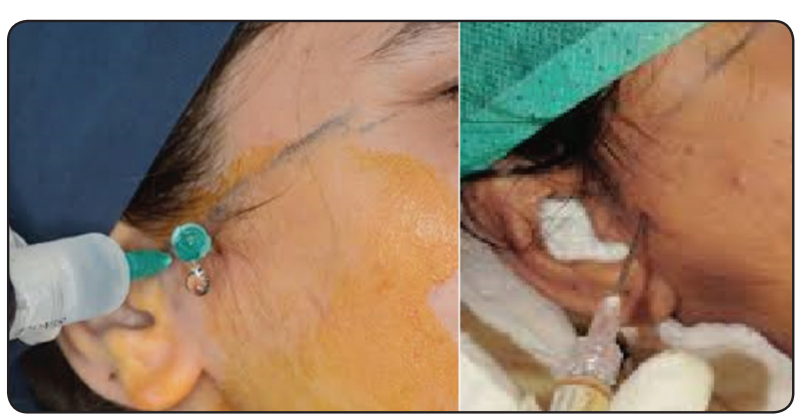

Fig. (2) Showing the arthrocentesis procedure and the injectable PRF injection in a sample of our cases.

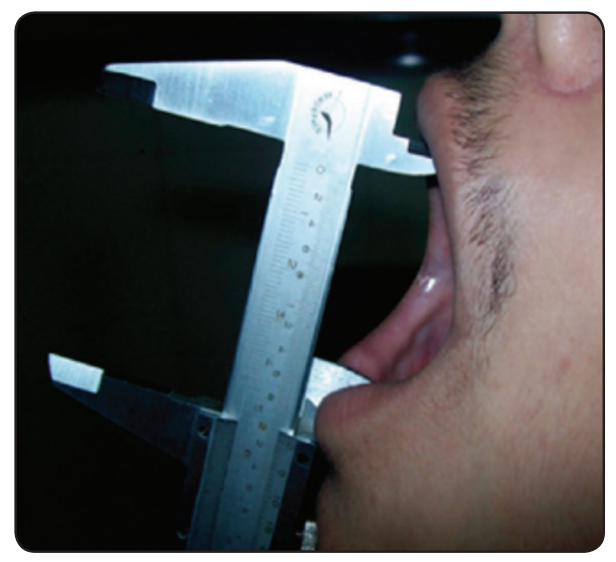

Fig. (3) Showing maximum mouth opening recording method 


\section{RESULTS}

Tables 2 and 3 summarize demographic characteristics of both groups. There is no significant difference in either age or duration of symptoms between both groups.

\begin{tabular}{|l|c|c|c|c|}
\hline \multirow{2}{*}{ TABLE (2) } & \multicolumn{2}{|c|}{ Group A } & \multicolumn{2}{c|}{ Group B } \\
\cline { 2 - 5 } & Mean & St Dev & Mean & St Dev \\
\hline Patient Age (Years) & 29 & 6.5 & 30 & 8 \\
\hline $\begin{array}{c}\text { Duration (months) } \\
\text { of symptoms }\end{array}$ & 20 & 12 & 16 & 7 \\
\hline
\end{tabular}

TABLE (3)

\begin{tabular}{|c|c|c|}
\hline Sex (gender) & Group A & Group B \\
\hline Males & 6 & 5 \\
\hline Females & 12 & 13 \\
\hline
\end{tabular}

\section{Clinical results}

\section{TMJ pain}

The evaluation of the TMJ pain during the study intervals is shown in table 4 and chart 1 . There is significant reduction in pain intensity in both groups after 2 and 4 weeks. After 8 weeks pain score is "zero" in both groups. Where the group B cases reported better relief in the pain test compared to those of group A.

There is no statistically significant difference between both groups preoperatively and after 2 and 4 weeks.

TABLE (4)

\begin{tabular}{|c|c|c|c|c|c|}
\hline \multirow{2}{*}{ Pain } & \multicolumn{2}{|c|}{ Group A } & \multicolumn{2}{c|}{ Group B } & \multirow{2}{*}{ P value } \\
\cline { 2 - 5 } & Mean & St Dev & Mean & St Dev & \\
\hline Preoperative & 8 & 1.5 & 8 & 1.5 & 0.373 \\
\hline 2 weeks & 5.5 & 1.5 & 3 & 2 & 1.686 \\
\hline 4 weeks & 1.5 & 1.5 & 0.5 & 0.5 & 1.233 \\
\hline 8 weeks & 0.5 & 0.5 & 0 & 0 & \\
\hline
\end{tabular}

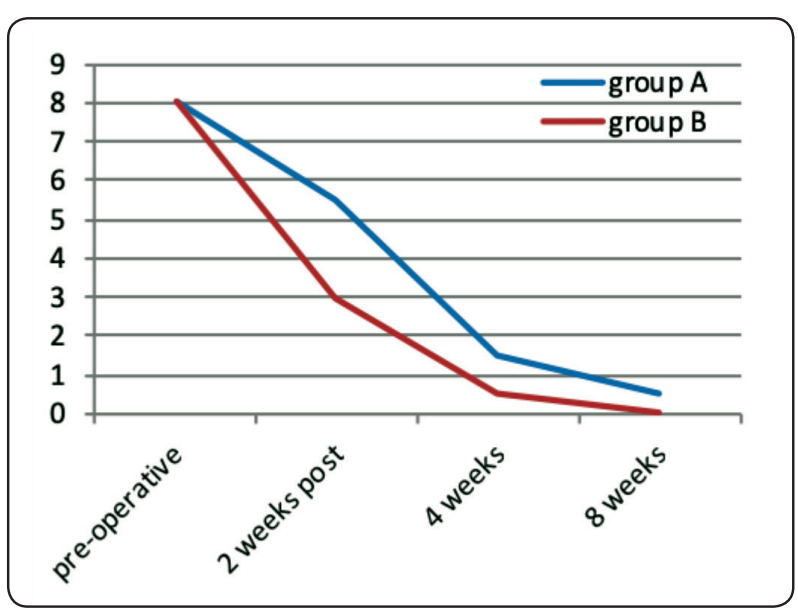

Chart (1) Demonstrating the flow of pain reduction in both groups.

\section{Maximum mouth opening}

Table 5 review the assessment of maximum mouth opening at the study intervals. PRF injection group (B) showed observed increase in the MMO from $25.58 \pm 3.18 \mathrm{mms}$ pre operative to $37.5 \pm 6.4$ immediately postoperative and continued to rise reaching an average $44.08 \pm 4.17 \mathrm{mms}$ only after 2 weeks denoting a rapid return to full range of mouth opening. Then followed by insignificant increase along the 4 and 8 weeks intervals.

In the Arthrocentesis group, there is noted increase in the MMO immediately postoperative averaging $36.75 \pm 1.91 \mathrm{mms}$ then followed by an insignificant increase at the 2 weeks interval but then begin to improve at the 4 and 8 weeks followups and finishing at an average MMO of $39.58 \pm 2.39 \mathrm{mms}$.

Statistical evaluation of both groups results denoted statistically nonsignificant results when comparing each interval alone, however comparing the full results of group A with group B revealed a $\mathrm{p}$ value of 0.0437 which denotes statistically significant improvement of the injectable PRF group MMO. 
TABLE (5)

\begin{tabular}{|c|c|c|c|c|c|}
\hline \multirow{2}{*}{ MMO } & \multicolumn{2}{|c|}{ Group A } & \multicolumn{2}{|c|}{ Group B } & \multirow{2}{*}{$P$ value } \\
\hline & Mean & St Dev & Mean & St Dev & \\
\hline Preoperative operative & 25.7 & 2.7 & 25.5 & 3.1 & 0.137 \\
\hline Immediate post operative & 36.7 & 1.9 & 37.5 & 6.4 & 0.130 \\
\hline 2 weeks & 39.2 & 2 & 44 & 4.1 & 3.591 \\
\hline 4 weeks & 39.5 & 2.3 & 45 & 3.5 & 4.470 \\
\hline 8 weeks & 39.8 & 2.1 & 45.5 & 3.9 & 4.403 \\
\hline
\end{tabular}

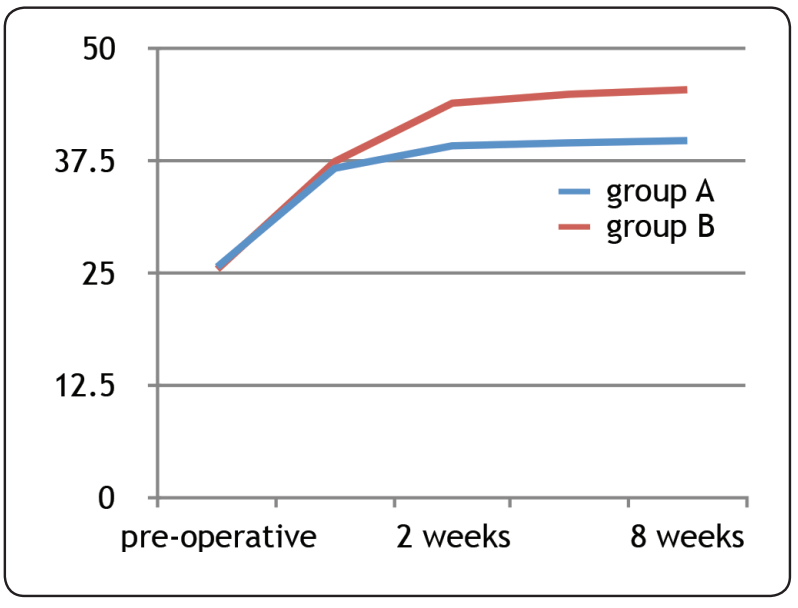

Chart (2) Demonstrating the range of MMO along the follow up intervals of both groups measured in mms.

\section{Clicking}

Table 6 demonstrates the frequency of joint clicking in both groups throughout the follow up intermissions. There is no statistically significant difference preoperatively. Immediately postop the frequency of joint clicking in the Arthrocentesis group is statistically significantly lower than in the PRF injection group. This was persistent till 4 weeks postoperatively. 8 weeks postoperatively, there is no statistically significant difference between both groups.

\begin{tabular}{|c|c|c|}
\hline \multirow{2}{*}{ TABLE (6) } & \multicolumn{2}{|c|}{ No of patients with clicking } \\
\cline { 2 - 3 } & Group A & Group B \\
\hline Preoperative & 18 & 18 \\
\hline Immediate & 9 & 4 \\
\hline 2 Weeks & 9 & 3 \\
\hline 4 Weeks & 8 & 2 \\
\hline 8 Weeks & 2 & 1 \\
\hline
\end{tabular}

\section{DISCUSSION}

Injectable PRF has been utilized in various clinical situations in order to regenerate tissues. It is becoming a valuable adjunct to promote healing in many procedures in dental and oral surgery such as periodontal regeneration in dental implants, ${ }^{(23)}$ bone regeneration in grafts $^{(24)}$, and fractures ${ }^{(25)}$. In a retrospective study, Taschieri et $\mathrm{al}^{(26)}$ assessed the soft tissue healing, implant and prosthesis survival, marginal bone level changes and biological complications of immediate postextraction implants with or without the use of pure platelet-rich plasma (P-PRP). They found that the P-PRP implant group showed a better soft tissue healing in the first 7 days after surgery compared to non-P-PRP implant group. However, in the medium-term follow-up, the two groups showed equivalent clinical and radiographic outcomes.

Considering the fact that the primary aim of treatment is to relieve pain and to restore complete range of mandibular motion rather than removal 
of the cause, arthrocentesis has been assessed as a treatment for disc displacement with reduction which is stubborn to conservative therapies and present data suggest that it is likely an effective therapy

Thus, this study was designed with an aim to evaluate the efficacy of intra-articular injection of PRF for treatment of DDWR in comparison to conventional arthocentesis. In the present study, there were no significance differences between the two groups in age, gender, duration of symptoms and evaluation of the investigated parameters in baseline. This is important as part of the statistical protocol. Also, the establishment of specific inclusion criteria is important when judging results.

In the current study, both groups showed significant improvement in TMJ pain, MMO and clicking 2 weeks after treatment that maintained thereafter. Considering that increased concentrations of inflammatory mediators have been identified in the synovial fluid of patients with internal derangement, suggesting an underlying degenerative or inflammatory process ${ }^{(28)}$.The excellent patient response to Arthrocentesis could be attributed to the disruption of adhesions, and washing out of inflammatory mediators resulting in decreased pain and increased range of motion ${ }^{(29-31)}$. On the other hand, efficacy of injectable PRF may be related to its anti-inflammatory effect as evident by the observations of Lippross et al. ${ }^{(32)}$ In their experimental study in a pig model of rheumatoid arthritis of the knee joint, interleukin-1, interleukin-6, tumor necrosis factor, vascular endothelial growth factor, and insulin-like growth factor 1 protein content was measured by immunoassay before and 2 weeks after the second intra-articular injection of normal saline or PRP. They found that the tissue concentrations of the tested inflammatory markers returned to control levels when injectable PRF was used, and that the reduction of each protein was significant compared to levels in the saline injected specimens.
The results of the current study highlights once again the usefulness of arthrocentesis for treatment of patients with disc displacement with reduction (DDWD) and complement the results of other studies. Also, our results are in general agreement with other studies that proved the efficacy of intraarticular injection of PRF as a treatment for painful TMJ. However, studies into its clinical efficiency are not conclusive and one of the main reasons for this is that different PRF preparations are used, eliciting different responses that cannot be compared ${ }^{(29-34)}$

Of these different reactions is the effect of intraarticular injection of PRF on TMJ clicking. Four weeks after PRF injections, the number of patients with clicking decreased from 12 to 8 in the present study. Contrary, Moon et al ${ }^{(36)}$ found no progress in 4 patients with TMJ clicking at the same interval. This in part may be due to their small sample size.

Comparing the two groups in the present study revealed more beneficial effects of PRF injection after arthrocentesis (group B) than arthrocentesis alone on MMO and joint sound. The improvement in MMO at 2, 4 and 8 weeks after treatment as well as in TMJ sounds immediately postoperative and at 2 and 4 weeks after treatment is markedly higher in the PRF group than in the arthrocentisis group. The difference in pain intensity between two groups was not statistically significant. These findings weren't contradicting with those of Hanc et al ${ }^{(27)}$ who found that pain intensity and joint sound was statistically significant improved in their PRP group more than in the arthrocentesis group and that the difference in MMO between the two groups was not statistically significant.

\section{CONCLUSION}

Intra-articular PRF injection after arthrocentesis is an effective treatment method over arthrocentesis (joint lavage) alone for the management of the TMJ internal derangements. 


\section{REFERENCES}

1. Ufuk Tatli and Vladimir Machon. Internal Derangements of the Temporomandibular Joint: Diagnosis and Management, Temporomandibular Joint Pathology - Current Approaches and Understanding, Yusuf Emes, Buket Aybar and Gühan Dergin, IntechOpen, DOI: 10.5772/intechopen.72585. 2017.

2. Wilkes $\mathrm{CH}$. Internal derangements of the temporomandibular joint. Pathological variations. Archives of Otolaryngology - Head \& Neck Surgery. 115:469-477,1989.

3. Schiffman E, Ohrbach R, Truelove E, Look J, Anderson G, Goulet JP, List T, Svensson P, Gonzalez Y, Lobbezoo F, Michelotti A, Brooks SL, Ceusters W, Drangsholt M, Ettlin D, Gaul C, Goldberg LJ, Haythornthwaite JA, Hollender L, Jensen R, John MT, De Laat A, de Leeuw R, Maixner W, van der Meulen M, Murray GM, Nixdorf DR, Palla S, Petersson A, Pionchon P, Smith B, Visscher CM, Zakrzewska J, Dworkin SF, International RDC/TMD Consortium Network, International association for Dental Research; Orofacial Pain Special Interest Group, International Association for the Study of Pain. Diagnostic criteria for temporomandibular disorders (DC/TMD) for clinical and research applications: Recommendations of the International RDC/TMD Consortium Network and Orofacial Pain Special Interest Group. Journal of Oral \& Facial Pain and Headache. 28:6-27,2014.

4. Manfredini d, Marini M, Pavan M, Pavan 1, Guarda-nardini 1 Psychosocial profiles of painful TMD patients. J Oral Rehabil. 36(3): 193-198, 2009.

5. Dolwick MF. The role of temporomandibular joint surgery in the treatment of patients with internal derangement. Oral Surg Oral Med Oral Pathol Oral Radiol Endod. 83(1): 150-155, 1997.

6. Nitzan DW, Price A. The use of arthrocentesis for the treatment of osteoarthritic temporomandibular joints. J Oral Maxillofac Surg. 59(10): 1154-1159, 2001.

7. Sharma SM, Thakar D.The Clinical Efficacy of Autologous Concentrated Platelets in Treatment of Tmj Disorders - a pilot study, 4(3): 70-74, 2014.

8. Anitua E. Plasma rich in growth factors: preliminary results of use in the preparation of future sites for implants. Int J Oral Maxillofac Implants. 14(4): 529-535, 2018.

9. Hassan EF, Ali TM, Abdulla NS. The Clinical Efficiency of Platelet Rich Plasma in the Treatment of Temporomandibular Joint Disorders. 41: 226-231, 2016.
10. Pihut M, Szuta M, Ferendiuk E, Ze DN. Evaluation of Pain Regression in Patients with Temporomandibular Dysfunction Treated by Intra-Articular Platelet-Rich Plasma Injections: A Preliminary Report. 2014.

11. Choukroun J, Ghanaati S. Reduction of relative centrifugation force within injectable platelet-rich-fibrin (PRF) concentrates advances patients' own inflammatory cells, platelets and growth factors: the first introduction to the low speed centrifugation concept. Eur J Trauma Emerg Surg. 44(1): 87-95, 2018.

12. El Bagdadi K, Kubesch A, Yu X, Al-Maawi S, Orlowska $A$, et al. Reduction of relative centrifugal forces increases growth factor release within solid platelet-rich-fibrin (PRF)-based matrices: a proof of concept of LSCC (low speed centrifugation concept). 2017.

13. Miron RJ, Fujioka-kobayashi M, Hernandez M. Injectable platelet rich fibrin (i-PRF): opportunities in regenerative dentistry ? Clin Oral Investig. 21(8): 2619-2627, 2017.

14. Albilia JB DMD, MSc, Herrera-Vizcaino C DDS, Weisleder H BSc, Choukroun J DM, Ghanaati S MD, DMD, PhD. Liquid platelet-rich fibrin injections as a treatment adjunct for painful temporomandibular joints: preliminary results. Cranio. Sep 20:1-13, 2018.

15. Menerva A Elgazzaz, Fatma A khalifa, Ghada A khalifa and Shadia A Elsayed. The Use of Injectable Platelet Rich Fibrin in theTreatment of Tempromandibular Joint Hypermobility. ADJ-for Girls (6), No. 4; 507:515, October 2019.

16. Arafat SW, Chehata IM. Clinical and biochemical assessment of different injection materials following arthrocentesis for the treatment of internal derangement of the temporomandibular joint: A comparative study. Tanta Dent J. 13:102-8, 2016.

17. Farah Taha and Ameena Diajil. Effectiveness of Intra articular Injection of Platelet-Rich Plasma in Patients with Anterior Disc Displacement with Reduction. J Bagh College Dentistry. vol 29 (1), 2017.

18. Girish Giraddi, A. Siddaraju, Bimleshwar Kumar and Chandravir Singh. Internal Derangement of Temporomandibular Joint: An Evaluation of Effect of Corticosteroid Injection Compared with Injection of Sodium Hyaluronate after Arthrocentesis. Journal of maxillofacial and oral surgery. 11; 258-63, 2012.

19. Varela, Hugo \& Souza, Júlio \& Nascimento, Rubens \& Júnior, Raimundo \& Vasconcelos, Roseane \& Cavalcante, Rômulo \& Guedes, Paulo \& Araújo, Aurigena. Injectable platelet rich fibrin: cell content, morphological, and protein characterization. Clinical Oral Investigations. Mar; 23(3):1309-1318, 2019. 
20. Del Corso M, Dohan Ehrenfest DM. Immediate implantation and peri-implant Natural Bone Regeneration (NBR) in the severely resorbed posterior mandible using Leukocyte-and Platelet-Rich Fibrin (L-PRF): a 4-year follow up. http://asnanportal.com/images/dental_report/periodonticsoral surgeryimplants/poseido-201312109-16-delcor-copia. pdf. 2013.

21. C. Herrera-Vizcaíno, E. Dohle, S. Al-Maawi, P. Booms, R. Sader, C.J. Kirkpatrick, J. Choukroun and S. Ghanaati. Platelet rich fibrin secretome incuces three dimentional angiogenic activation in vitro. European Cells and Materials. 37; 250-264, 2019.

22. Mourão, Carlos \& Valiense, Helder \& Melo, Elias \& Mourão, Natália \& Maia, Monica. Obtention of injectable platelets rich-fibrin (i-PRF) and its polymerization with bone graft: technical note. Revista do Colégio Brasileiro de Cirurgiões. 42. 421-423, 2015.

23. Plachokova, A.S., D. Nikolidakis, J. Mulder, J.A. Jansen, and N.H. Creugers, Effect of platelet-rich plasma on bone regeneration in dentistry: a systematic review. Clin Oral Implants Res. 19(6); 539-45, 2008.

24. Birang, R., A. Torabi, M. Shahabooei, and M. Rismanchian, Effect of plasma-rich in platelet-derived growth factors on peri-implant bone healing: An experimental study in canines. Dent Res J (Isfahan). 9(1); 93-9. 2012.

25. Qiu, J., C. Zhang, Y. Guo, T. Yuan, and Z. Xie, [Clinical study on PRP in improving bone repair]. Zhongguo Xiu Fu Chong Jian Wai Ke Za Zhi. 23(7); 784-7, 2009

26. Taschieri, S., A. Lolato, M. Ofer, T. Testori, L. Francetti, and M. Del Fabbro, Immediate post-extraction implants with or without pure platelet-rich plasma: a 5-year followup study. Oral Maxillofac Surg. 21(2); 147-157, 2017.

27. Hanc M, Karamese M, Tosun Z, Aktan T, Duman S, SavaciN.Intra-articular platelet-rich plasma injection for the treatment of temporomandibular disorders and a comparison with arthrocentesis.JCraniomaxillofac Surg. 43;16216,2015 .
28. Matsumoto K, Honda K, Ohshima M, Yamaguchi Y, Nakajima I, Micke P, Otsuka K. Cytokine profile in synovial fluid from patients with internal derangement of the temporomandibular joint: a preliminary study. Dentomaxillofac Radiol. 35(6);432-441, 2006.

29. Guarda-Nardini L et al, effects of arthrocentesis in the management of signs and symptoms of painful TMJ disc displacement with reduction. A pilot study, Oral Maxillofac Surg . 29-34, 2010.

30. Nitzan D, Dolwick M, Martinez G. Temporomandibular joint arthrocentesis: a simplified treatment for severe, limited mouth opening. J Oral MaxillofacSurg. 49; 1163 1167,1991 .

31. Shailesh Kumar et al, Temporomandibular Joint Arthrocentesis: A Prospective Study and Audit of 500 Joints of Central India .J Int Soc Prev Community Dent. 8(2); 124129, 2018.

32. Lippross S, Moeller B, Haas H, Tohidnezhad M, Steubesand N, Wruck CJ, et al: Intraarticular injection of plateletrich plasma reduces inflammation in a pig model of rheumatoid arthritis of the knee joint. Arthritis Rheum. 63(11); 3344-3353,2011.

33. Serindere G et al ,The effect of arthrocentesis in temporomandibular joint internal derangement with magnetic resonance imaging . International Journal of Applied Dental Sciences. 3(4); 148-151,2017.

34. Cerza F, Carnì S, Carcangiu A, Di Vavo I, Schiavilla V, Pecora, A.Comparison between hyaluronic acid and platelet-rich plasma, intra-articular infiltration in the treatment of gonarthrosis. Am. J. Sports Med. 40; 822-827,2012.

35. Pihut M, Szuta M, Ferendiuk E, Zeńczak,Więckiewicz D. Evaluation of Pain Regression in Patients with Temporomandibular Dysfunction Treated by Intra-Articular Platelet-Rich Plasma Injections: A Preliminary Report.Bio Med Research International. 14;132-169,2014.

36. Moon S, Lee S, Ryu J.Ultrasound-guided Platelet-rich Plasma Prolotherapy for Temporomandibular Disorders.J Oral Med Pain. 39; 140-145,2014. 